\title{
A temperature correlation for the radiation resistance of a thick-walled circular duct exhausting a hot gas
}

\author{
J. R. Mahan, ${ }^{a)}$ J. G. Cline, ${ }^{\text {b) }}$ and J. D. Jones ${ }^{\text {) }}$ \\ Virginia Polytechnic Institute and State University, Blacksburg, Virginia 24061
}

(Received 5 June 1983; accepted for publication 2 October 1983)

\begin{abstract}
It is often useful to know the radiation impedance of an unflanged but thick-walled circular duct exhausting a hot gas into relatively cold surroundings. The reactive component is shown to be insensitive to temperature, but the resistive component is shown to be temperature dependent. A temperature correlation is developed permitting prediction of the radiation resistance from a knowledge of the temperature difference between the ambient air and the gas flowing from the duct, and a physical basis for this correlation is presented.
\end{abstract}

PACS numbers: 43.20.Mv, 43.20.Rz, 43.85.Bh

\section{INTRODUCTION}

It is often desirable to know the radiation impedance at the end of a circular duct, such as an exhaust pipe or chimney, from which a hot gas flows into relatively cold surroundings. This quantity is useful for predicting the sound pressure level in the farfield of exhaust systems, and for assessing the potential for unstable combustion in industrial burners.

Measurements of the radiation impedance made under these conditions and reported in Refs. 1 and 2 indicate that the imaginary component, the reactance, is insensitive to temperature, but that the real part, the resistance, increases with the temperature of the gas flowing from the duct. No attempt was made in the studies cited to correlate the results obtained. The present paper presents a correlation, based on data from these studies as well as on new data obtained for this purpose, which permits the radiation resistance to be predicted for a wide range of differences between the temperature of the gas flowing from the duct and that of the surrounding air. Also presented is a physical basis for the observed variation of the radiation resistance with this temperature difference.

\section{REVIEW OF PREVIOUS STUDIES}

References 1 and 2 concern attempts to determine the extent to which the radiation impedance is influenced by temperature gradients near the open end of a duct. Fricker and Roberts ${ }^{1}$ used the classical impedance tube method to measure the radiation impedance for a circular duct whose ratio of inside diameter to outside diameter was 0.6 . The gas temperature in the duct ranged from $18^{\circ} \mathrm{C}-1000^{\circ} \mathrm{C}$, and the surrounding air temperature was maintained at $18^{\circ} \mathrm{C}$. The flow velocity in the duct was too low $(10 \mathrm{~m} / \mathrm{s}, \mathrm{M}<0.03)$ to

\footnotetext{
a) Associate Professor of Mechanical Engineering; presently Professor of Mechanical and Aerospace Engineering, West Virginia University, Morgantown, WV 26506.

${ }^{b)}$ Graduate Research Assistant; presently Engineer, General Dynamics Corporation, Fort Worth, TX.

c) Graduate Research Assistant; presently Instructor of Mechanical Engineering.
}

significantly influence the radiation impedance.

Measurements were obtained for the case of a uniform gas temperature in the duct as well as for the case of axial temperature gradients. When there was a significant temperature gradient, the pressure standing wave pattern in the duct was distorted. In this case, values of the standing wave ratio measured at several locations along the duct were extrapolated to the open end. However, there is no indication that a similar correction was applied to the wavelength. Because both the standing wave ratio and wavelength at the open end of the duct are needed to compute the radiation impedance at a given frequency, the lack of a suitable wavelength correction might be expected to compromise the results obtained.

The acoustic environment of the apparatus used in Ref. 1 is not described, nor are many details given concerning the manner in which the data were obtained. It is simply stated that the acoustic pressure probe, a small diameter stainless steel tube, could be positioned accurately in the duct. The possibility of thermal effects on the functioning of this probe is not discussed. If the probe was in a state of thermal equilibrium each time an acoustic pressure measurement was made, its temperature, and thus its sensitivity, would have been different depending on its depth of penetration into the hot duct. Of course, if the probe was not in a state of thermal equilibrium during the measurements, its sensitivity would have varied during the measurements and the values then would have depended on when they were obtained.

Fricker and Roberts' results are presented as graphs of the real and imaginary parts of the specific radiation impedance as a function of the product of the wavenumber and the inside radius of the duct. The wavenumber is defined with respect to the speed of sound based on the gas temperature at the duct exit. These results, which we obtained from Ref. 1 using a Numonics Corporation 1224 digitizing table, are reproduced in Fig. 1(a) and (b). The classical theory of $\mathrm{Ando}^{3}$ also appears in these figures. These measured impedances show considerable scatter, perhaps because the sensitivity of the acoustic pressure probe was not constant, as suggested above. In spite of this scatter, however, it is apparent that the real part of the radiation impedance, shown in Fig. 1(a), in- 


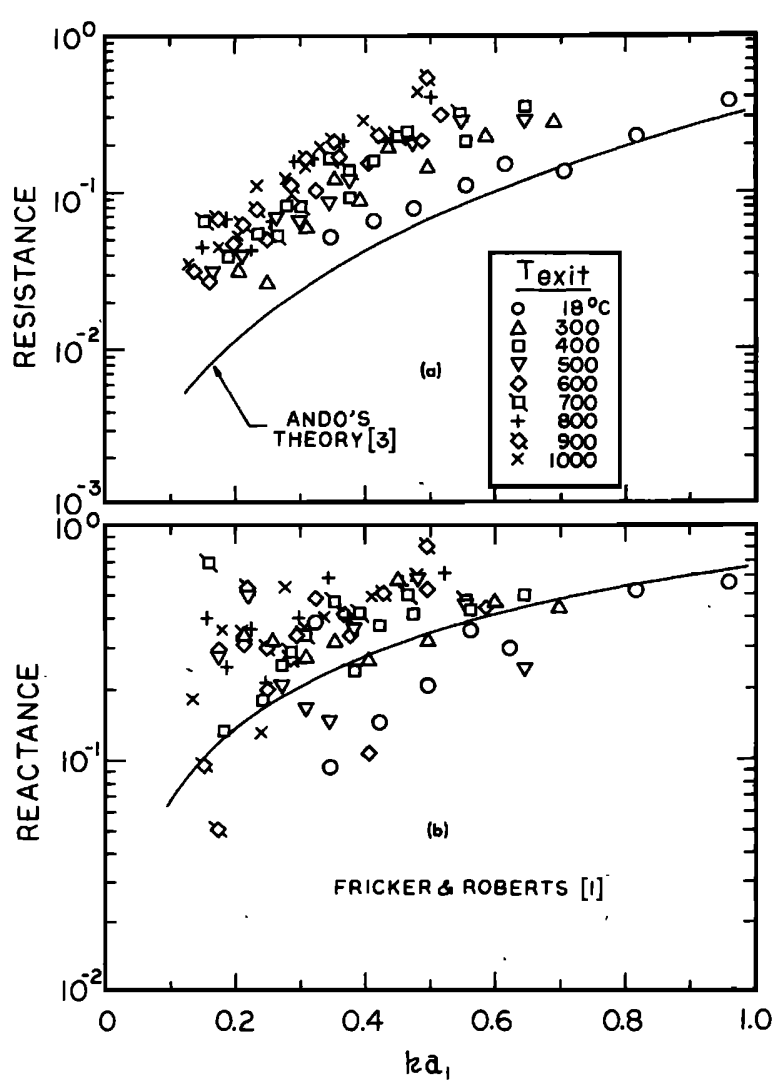

FIG. 1. Duct exit temperature dependence of the radiation impedance as reported in Ref. 1.

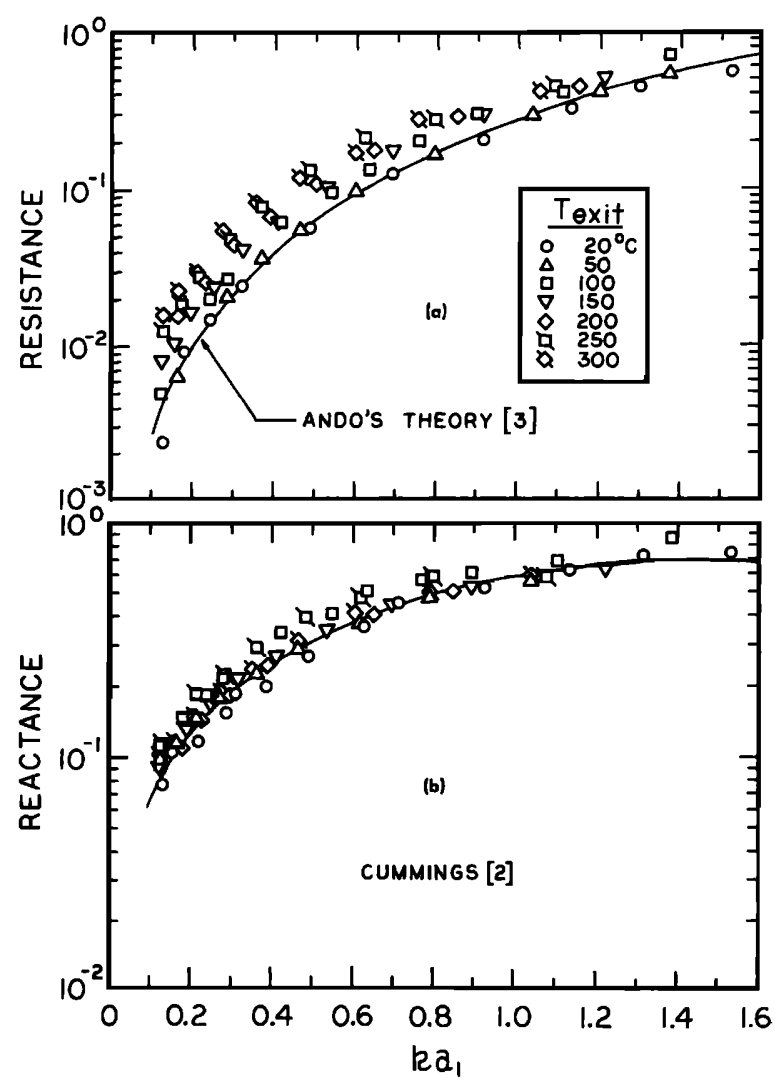

FIG. 2. Duct exit temperature dependence of the radiation impedance as reported in Ref. 2. creases with the gas temperature at the duct exit. There is no apparent temperature trend in the imaginary part of the radiation impedance [Fig. 1(b)]

Cummings ${ }^{2}$ also measured the radiation impedance of a thick-walled circular duct under circumstances similar to those of Fricker and Roberts, but at lower temperatures. The ratio of inside diameter to outside diameter for his apparatus was 0.81 . He was careful to insure that the temperature of his acoustic pressure probe, a double-walled glass tube, was always the same for each measurement. He tried to eliminate axial temperature gradients in the duct itself by selectively heating the duct walls.

Cummings' results are also presented as graphs of the real and imaginary parts of the specific radiation impedance as a function of a dimensionless wavenumber defined in terms of the gas temperature at the duct exit. His results, which we obtained from Ref. 2 using the Numonics digitizing table, are reproduced in Fig. 2(a) and (b). They show much less scatter than those of Fricker and Roberts, perhaps because of the care taken to maintain the acoustic pressure probe at a constant temperature. The theoretical results of Ando also appear in these figures. These measured impedances are in qualitative agreement with those of Fricker and Roberts in that the real part increases with temperature, while there is no apparent temperature trend in the imaginary part.

\section{PRESENT STUDY}

Our interest in this problem stems from a study of the acoustic source mechanisms in a long turbulent combustion chamber ${ }^{4}$. The radiation impedance of this combustion chamber was needed in order to recover its thermal-acoustic efficiency spectrum from the sound pressure spectrum in its farfield. The temperature range of interest for the study cited was limited to about $250^{\circ} \mathrm{C}$.

\section{A. Experimental apparatus and procedure}

The experimental apparatus used in the present study is shown in Fig. 3. It consists of a vertical Pyrex tube $1.0 \mathrm{~m}$ long and having inside and outside diameters of $\mathbf{7 4 . 6}$ and $\mathbf{7 9 . 8}$ $\mathrm{mm}$, respectively $\left(a_{1} / a_{2}=0.93\right)$. The lower end of the tube is connected to a small settling chamber and the upper end opens into the laboratory. A speaker located in the settling chamber and driven by an oscillator produces a standing wave in the tube at specified frequencies. Near the lower end of the tube is a small natural gas burner which can be displaced along the axis of the tube. Air enters the tube from the settling chamber and mixes with the natural gas, the mixture is burned, and the products of combustion exit from the open end.

The acoustic pressure in the tube downstream of the burner is measured by a probe borrowed from a B\&K 4002 impedance tube apparatus and modified to increase its reach. The probe must be introduced from the upper end of the tube because the burner occupies the axis on the other end. In order to protect the microphone from the hot gases flowing from the tube, an insulated plate 275- $\mathrm{mm}$ square is mounted normal to the tube axis $325 \mathrm{~mm}$ (about $4 \frac{1}{3}$ diam) 


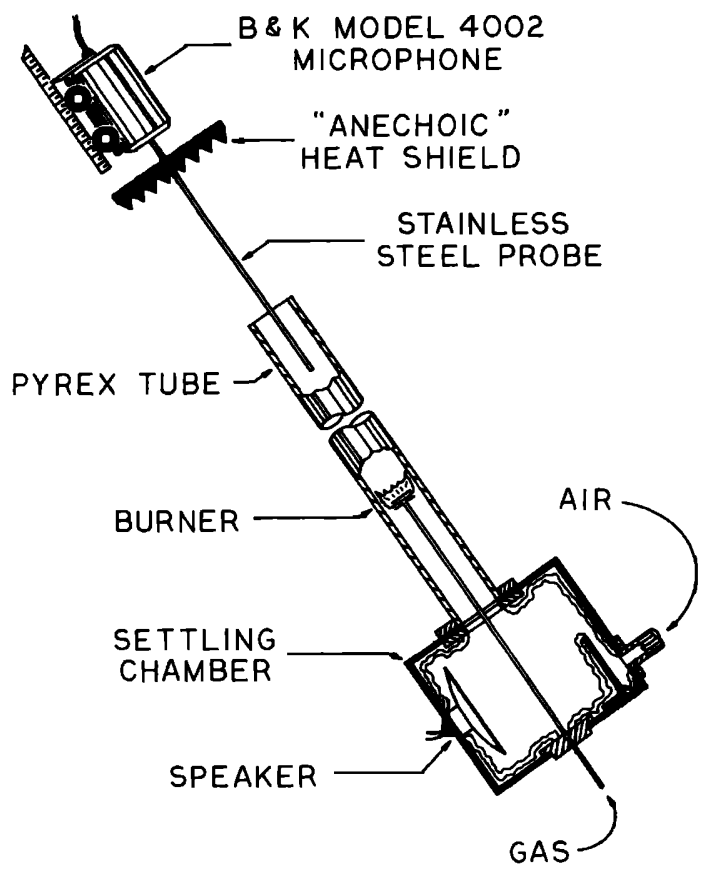

FIG. 3. Hot flow impedance tube apparatus used in the present study.

from the open end. The probe passes through a small hole in the center of this plate. The side of the plate which faces the open end of the tube is covered with an array of $6440-\mathrm{mm}$ deep glass wool wedges to reduce the reflection of acoustic energy back into the tube. As might be expected, the influence of this plate on the radiation impedance is clearly visible at low frequencies.

As explained in Sec. I, great care must be taken to assure that the temperature of the probe is constant during the measurements, and that the temperature is the same for each measurement. We accomplish this by plunging the probe rapidly into the tube and obtaining the pressure measurement before the temperature of the probe can change. After each measurement the probe is retracted and allowed to attain its original temperature before the next measurement. By indexing the final position of the probe along the axis of the tube for a series of such rapid insertions, an accurate standing wave pattern can be traced out for each frequency and heating condition of interest.

In addition to the local acoustic pressure on the axis of the tube, the temperature of the gas at the exit of the tube and in the laboratory, as well as the air and natural gas flows, are measured.

The classical impedance tube method consists of measuring the standing wave ratio near the open end of the tube and the axial position of the pressure node nearest the open end. These quantities are then used in relations given, for example, in Ref. 5 to compute the corresponding radiation impedance. This procedure supposes that the standing wave pattern is uniform throughout the tube. However, this is generally true only if the temperature of the gas in the tube is uniform. If there is a significant axial temperature gradient, the standing wave ratio and wavelength will vary along the tube, thus casting doubt on the validity of the method.
In order to establish the validity of the classical method in the presence of axial temperature gradients, at least for the limited range of heating conditions encountered in this study, we compare the pressure standing waves obtained as described above to those computed based on the values of radiation impedance measured in the classical way. The calculated waveforms are based on an acoustic propagation model which includes the effects of flow and axial temperature gradients. This model, the details of which are given in Ref. 6, is based on a first-order perturbation analysis of the unsteady one-dimensional flow of an inviscid ideal gas with heat loss to the walls. The resulting wave equations in acoustic pressure, particle velocity, and temperature are integrated starting at the open end of the tube with the measured value of pressure, the corresponding value of particle velocity based on the measured radiation impedance, and the corresponding isentropic acoustic temperature. In some cases the initial value of acoustic pressure may be varied slightly from the measured value to improve the agreement between the measured and calculated waveforms. This avoids according too much importance to a single element of a set of data.

The results of this comparison for a frequency of 1000 $\mathrm{Hz}$ and for a range of heating conditions characterized by different exit temperatures are given in Fig. 4. In each case the air entered the tube at a temperature of $17^{\circ} \mathrm{C}$ and with a mean velocity of $0.25 \mathrm{~m} / \mathrm{s}$, and the temperature in the laboratory was $17^{\circ} \mathrm{C}$. The thermal conditions in the tube were varied by changing the position and power level of the burner and, in one case, by thermally insulating the tube. The symbols in the figure represent the measured values of

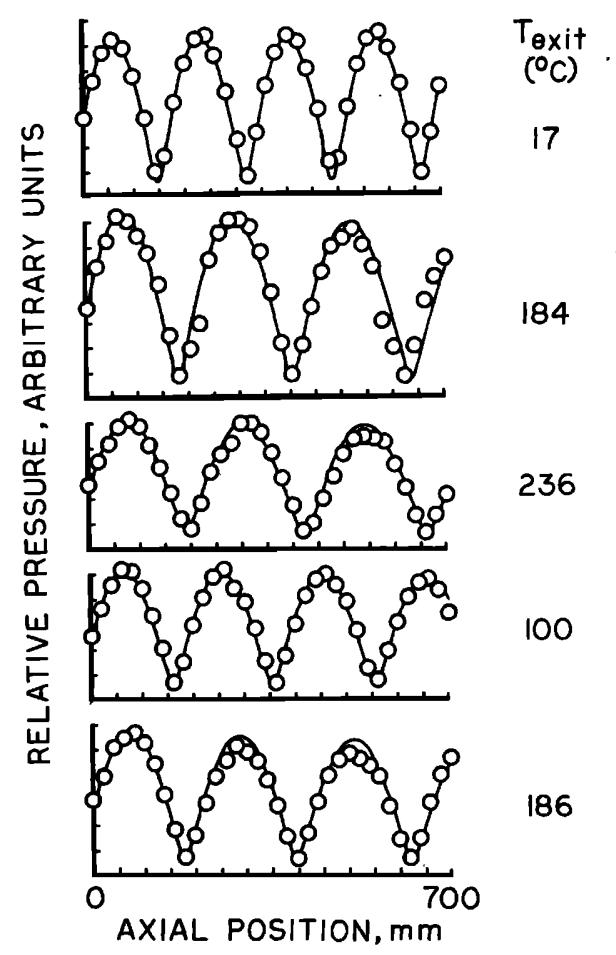

FIG. 4. Comparison of measured and computed pressure standing wave forms at $1000 \mathrm{~Hz}$ for the apparatus of Fig. 3 . 
acoustic pressure and the curves are the calculated waveforms. The excellent agreement between the experimental and calculated pressure standing wave patterns demonstrates that good values of the radiation impedance can be obtained by interpreting the data in the classical way without considering the distortion of the wave, at least for the limited temperature range of this study.

The real and imaginary parts of the radiation impedance obtained in the classical manner for the same heating conditions as in Fig. 4 are shown in Fig. 5(a) and (b), respectively. The dimensionless wavenumber $k a_{1}$ is based on the temperature of the gas at the tube exit and the inside radius of the tube. The impedances are in qualitative agreement
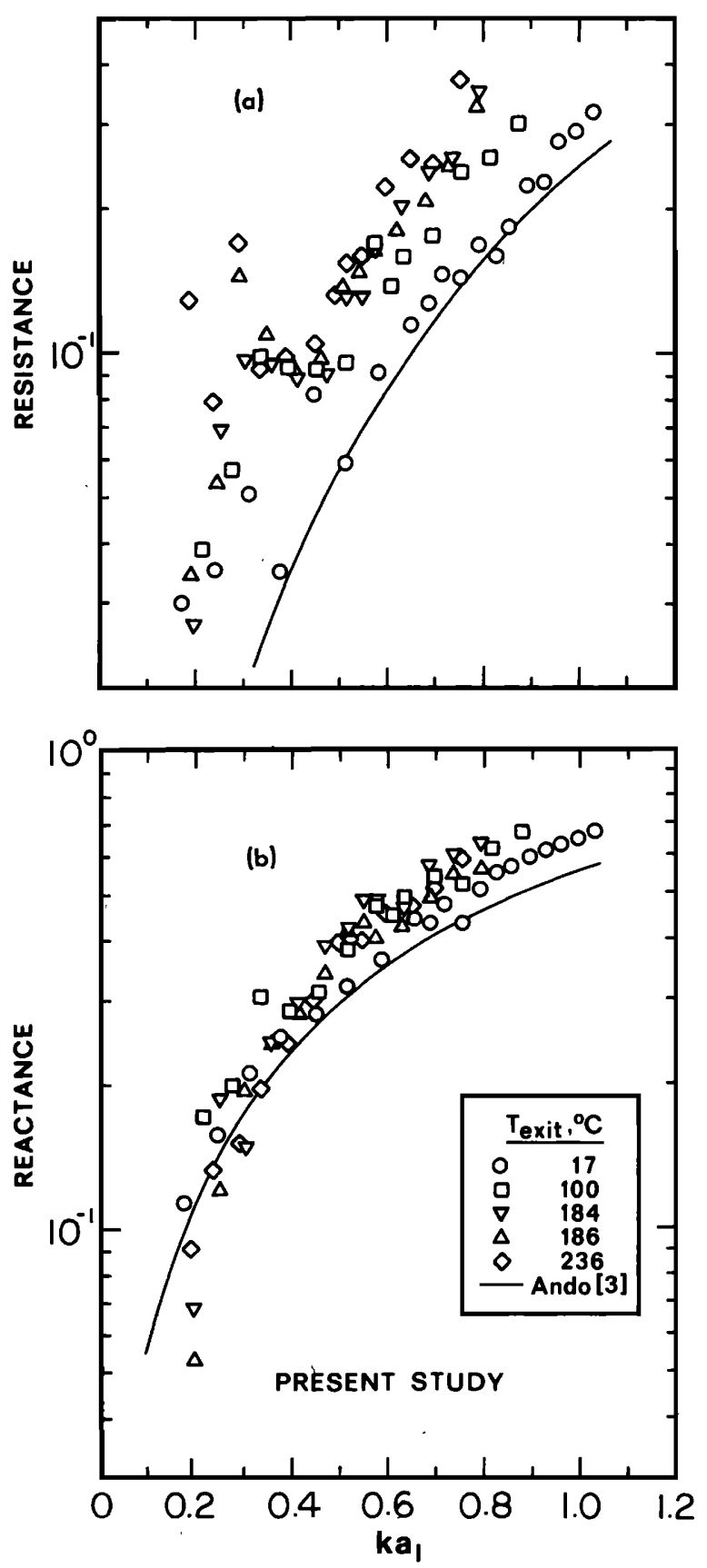

FIG. 5. Duct exit temperature dependence of the radiation impedance for the apparatus of Fig. 3. with those of Fricker and Roberts and of Cummings in that the real part increases with the temperature of the gas at the tube exit for a given dimensionless wavenumber, while the imaginary part is insensitive to this temperature.

\section{B. Interpretation of the results}

According to Ando's ${ }^{3}$ theoretical treatment of the radiation of sound from a semi-infinite, thick-walled circular duct into an anechoic space, the radiation impedance depends only on the Strouhal number $2 \pi f a_{1} / c$ for a given wall thickness. Of course, all real experiments involve finitelength ducts opening into spaces which, at least over a part of the frequency range, are not perfectly anechoic. The applicability of Ando's analysis is further limited in the present study by the presence of significant differences between the temperature of the gas flowing from the tube and that of the surrounding air. Thus it is a bit unrealistic to expect the Strouhal number and diameter ratio to correlate the data from these experiments. However, that is evidently what has happened, to within experimental uncertainty, in the case of the imaginary part of the impedance. It is probable that this occurs, as Cummings ${ }^{2}$ suggests, because the radiation reactance is mostly determined by the oscillation of a plug of fluid in the vicinity of the open end of the tube whose temperature is near that of the gas in the tube. The failure of the Strouhal number based on the temperature of the gas at the open end of the tube to collapse the radiation resistance data for a given diameter ratio indicates that the resistance is dominated by a mechanism whose characteristic temperature is different from the gas temperature in the tube.

As a starting point in our search for a temperature correlation, we note that when the base $10 \log$ of the specific resistance $\theta$ is plotted against the base $10 \log$ of the dimensionless wavenumber $k a_{1}$, there results a straight line; i.e., to a good approximation

$$
\log _{10}(\theta)=A+B \log _{10}\left(k a_{1}\right) \text {. }
$$

These are, in fact, the coordinates which Cummings uses to present his results in Ref. 2. The coefficients $A$ and $B$ in Eq. (1) might reasonably be expected to be functions of the temperature difference $T_{e}-T_{a}$, where $T_{e}$ is the duct exit gas temperature and $T_{a}$ is the ambient air temperature; and the radius ratio, $a_{1} / a_{2}$, where $a_{1}$ is the internal radius and $a_{2}$ is the external radius of the duct. Our approach then is to try to find the variations of the coefficients $A$ and $B$ with the temperature difference and radius ratio.

The data from Figs. 1(a), 2(a), and 5(a) are replotted using $\log -\log$ coordinates in Figs. 6, 7, and 8, respectively. The resistances at each temperature have been separated vertically in these figures in order to render them more readable. The straight lines are linear regressions of the form of Eq. (1) passed through the filled symbols. Ando's theory is shown as a dashed line in these figures. In each case the agreement is good between theory and experiment for the uniform temperature case.

The open symbol in Fig. 6 and the 25 open symbols in Fig. 8 have not been used to establish the regression coefficients because they clearly deviate from straight-line behavior. The open symbols in Fig. 8, which involves the resis- 


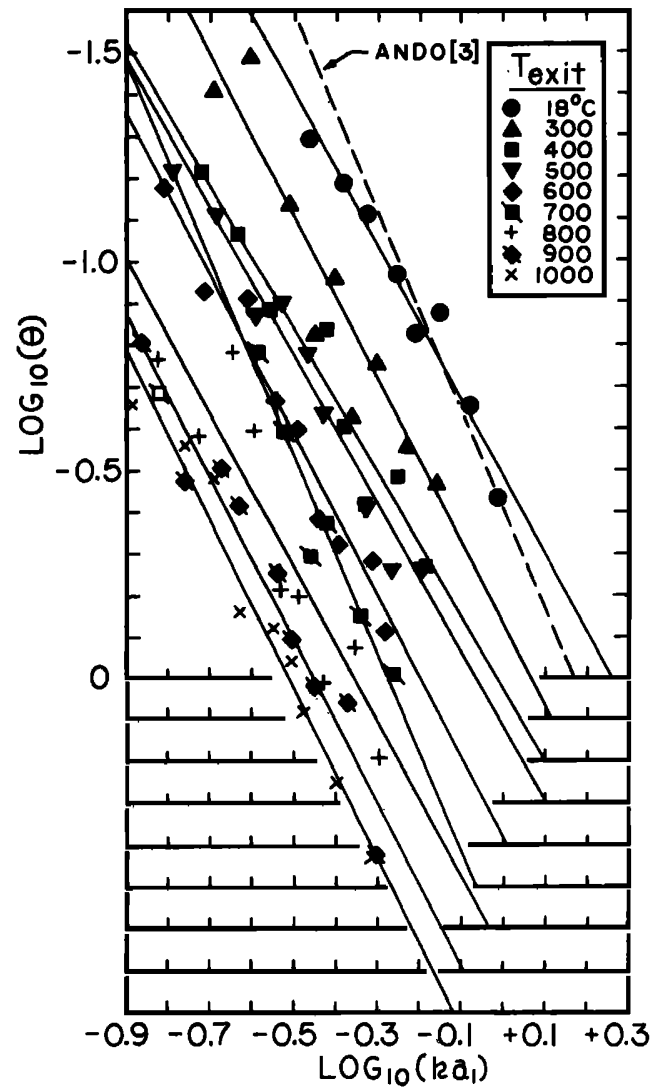

FIG. 6. Radiation resistances from Ref. 1 replotted on $\log$-log coordinates.

tances for the present experiment, correspond to low frequencies, $k a_{1}^{\prime}<0.45$, for which the radiation resistance is clearly altered by the presence of the plate provided to protect the microphone from the hot gas issuing from the tube.

Figure 9 is a plot of the intercept coefficient $A$ versus the temperature difference $T_{e}-T_{a}$ for the linear regressions of Figs. 6-8. The coefficients derived from Ando's theoretical curves corresponding to $T_{a}=T_{e}$ appear in this figure as filled symbols. The coefficients for all three sets of data increase monotonically with the temperature difference and generally follow the same trend. Nevertheless, it is possible to identify three distinct characteristics corresponding to the three data sets. This might be expected, of course; on the basis of the fact that the duct radius ratios are different in the three experiments. However, the slopes of the three characteristics in Fig. 9 do not seem to be related in any simple or explainable way to the radius ratios. The two experiments whose radius ratios are most nearly the same, that of Cummings $\left(a_{1} / a_{2}=0.81\right)$ and that of the present work $\left(a_{1}\right)$ $a_{2}=0.93$ ), produce trends in Fig. 9 which are the furthest apart. The third experiment, that of Fricker and Roberts, has a significantly smaller radius ratio $\left(a_{1} / a_{2}=0.60\right)$ but produces a trend in Fig. 9 which lies between those of the other two experiments. The problem with attempting a wall thickness correlation on the basis of these data is further illustrated in Fig. 10, which compares the experimental and theoretical variations of the intercept and slope coefficients with radius ratio for the case of $\Delta T=0$. Although the measured and predicted values of the intercept coefficient are in

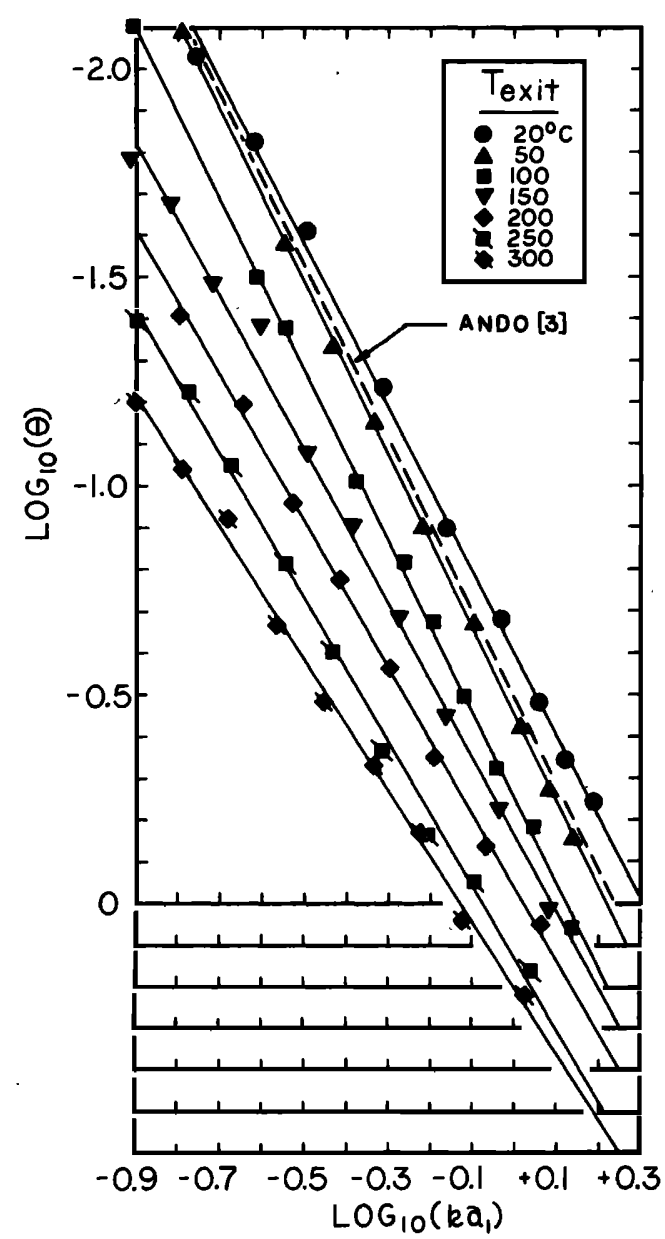

FIG. 7. Radiation resistances from Ref. 2 replotted on $\log -\log$ coordinates.

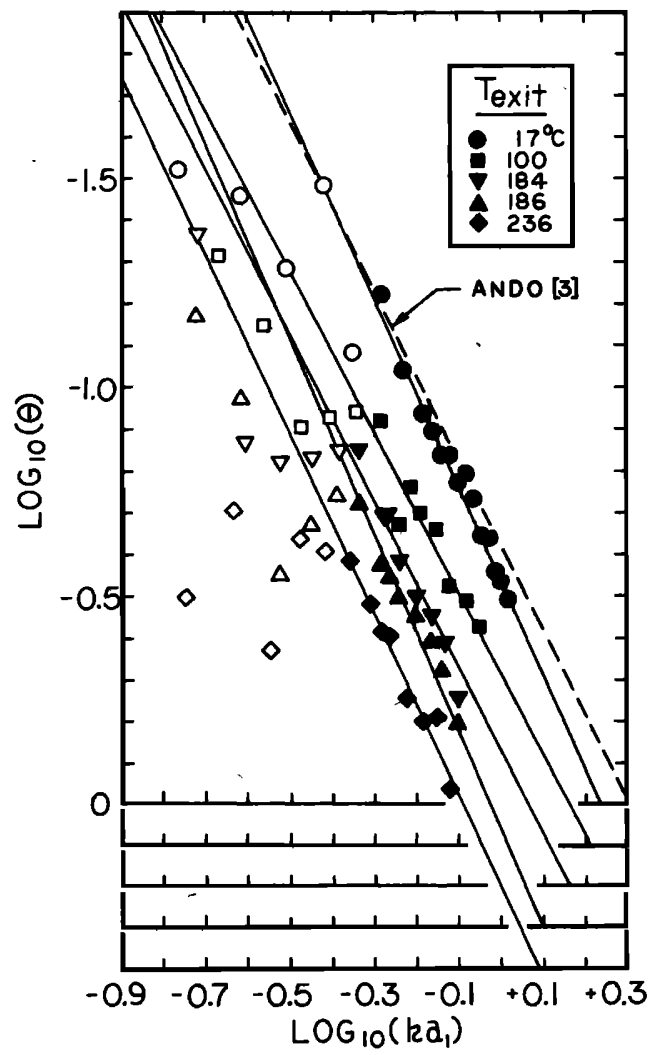

FIG. 8. Radiation resistances from the present study replotted on $\log -\log$ coordinates. 


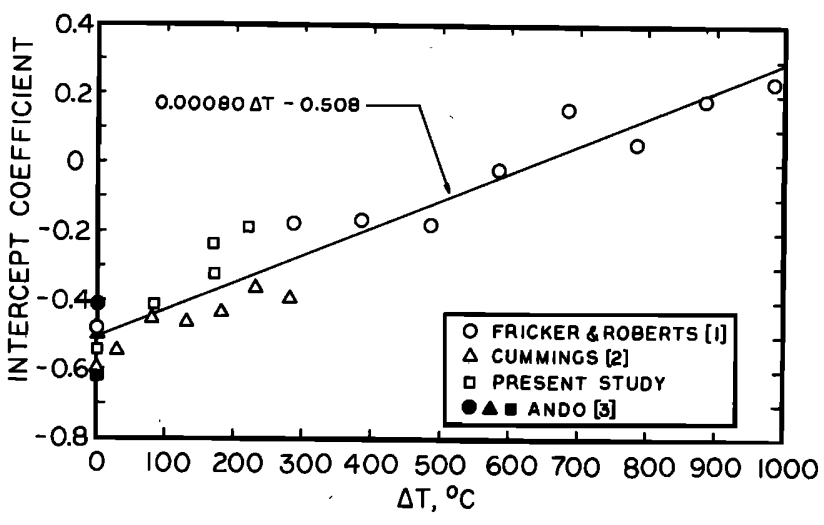

FIG. 9. Temperature dependence of the intercept coefficient $A$ of Eq. (1).

reasonable agreement, those for the slope coefficient diverge sharply. Evidently other differences among the three experiments, such as the duct lengths and acoustic properties of the surroundings, not addressed by Ando's idealized theory, are more important than the radius ratio in determining the radiation resistances in these experiments.

Insufficient information is given in Refs. 1 and 2 about the acoustic environment of the experimental apparatuses to justify a correlation based on any other parameters. Since there is also no consistent basis for a correlation based on the radius ratio, all of the intercept coefficients of Fig. 9 are then treated as a single data set for purposes of the temperature correlation. The straight line passing through these data is a linear regression of the form

$$
A=0.00080 \Delta T-0.508 \text {, }
$$

with a coefficient of determination $r^{2}$ of 0.91 .

The slope coefficient $B$ is plotted as a function of the temperature difference $T_{e}-T_{a}$ in Fig. 11. Some of the same observations can be made about this figure as were made about Fig. 9. The coefficients for the three data sets seem to

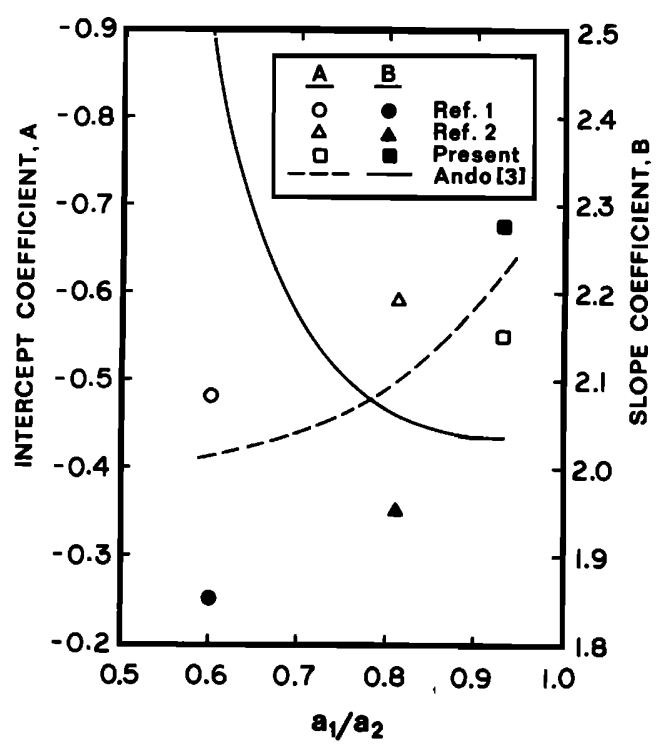

FIG. 10. Comparison of experimental and theoretical variations of the slope and intercept coeficients with radius ratio for the case $\Delta T=0$.

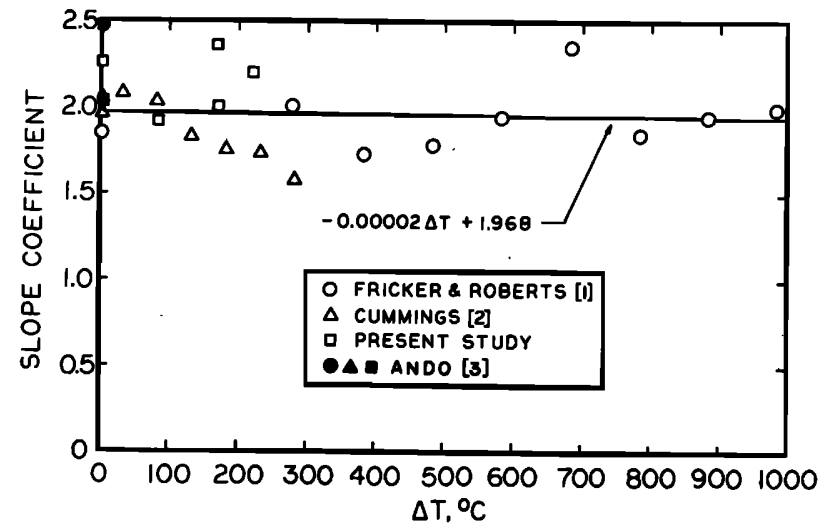

FIG. 11. Temperature dependence of the slope coefficient $B$ of Eq. (1).

follow patterns of their own while together defining a consistent trend. Also, the coefficients for the two experiments having similar radius ratios seem to diverge while the coeffcients for the third experiment, which has a significantly smaller radius ratio, fall between them. If these results are also treated as a single data set for purposes of the temperature correlation, there results a slope coefficient which is essentially independent of the temperature difference. The nearly horizontal straight line in the figure is a linear regression of the form

$$
B=-0.00002 \Delta T+1.968 \text {, }
$$

with a coefficient of determination of 0.008 . This low value of the determination coefficient reflects the dispersion of the data which occurs because the slope coefficient $B$ represents the first derivative of experimental data. Of course, differentiation of data always enhances any scatter already present. Note that the variation of the slope coefficient with temperature difference, given by Eq. (3), is sufficiently weak that it could be assigned a constant value equal to 1.960 in practice with very little error.

Equations (1) and (2) and the approximation for Eq. (3) can now be combined into a single relation which correlates all of the data from the three independent experiments repre-

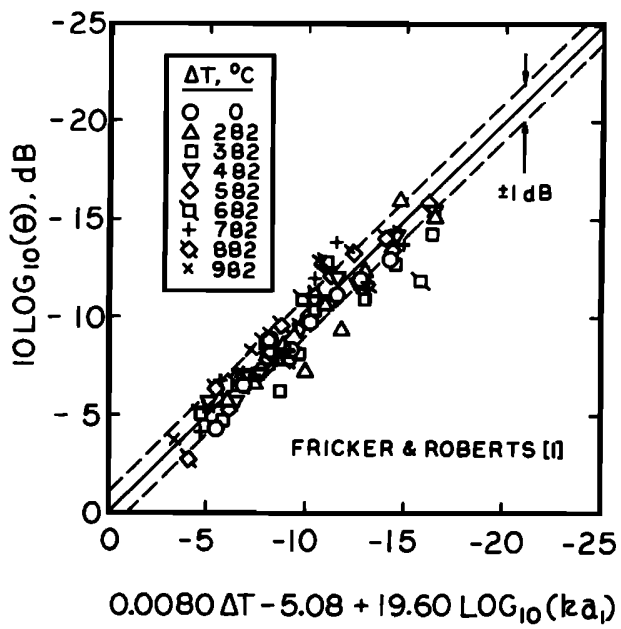

FIG. 12. Correlation of the radiation resistances from Ref. 1 using Eq. (4). 


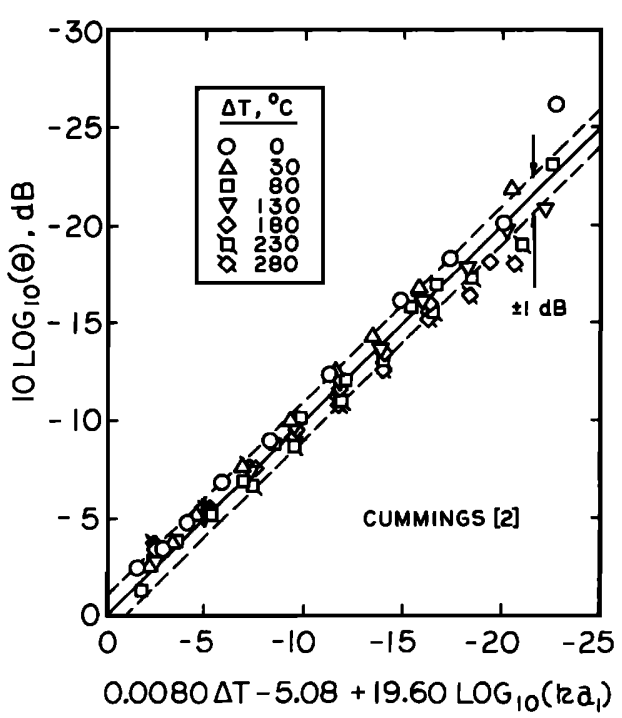

FIG. 13. Correlation of the radiation resistances from Ref. 2 using Eq. (4).

sented by the solid symbols in Figs. 6-8 as follows:

$\log _{10}(\theta)=0.00080 \Delta T-0.508+1.960 \log _{10}\left(k a_{1}\right)$.

All of the resistance data, including those represented by the open symbols in Figs. 6 and 8, are plotted against this correlation function in Figs. 12-14. On the basis of these figures, the correlation can be deemed successful, especially if one considers the scatter in the original data and the unsampled physical differences among the three experiments. Most of the measured resistances used to formulate the correlation (solid symbols in Figs. 6-8) fall within $\pm 1.0 \mathrm{~dB}$ of the values predicted by the correlation. The uncertainty associated with the lack of precise knowledge of the acoustic environment, to be expected in almost any practical application envisioned for the proposed correlation, could easily account for a $\pm 1.0-\mathrm{dB}$ deviation in the radiation resistance. Thus the scatter in Figs. 12-14 does not signal a serious limitation to the proposed correlation.

It is interesting to note in Fig. 14 that those data from

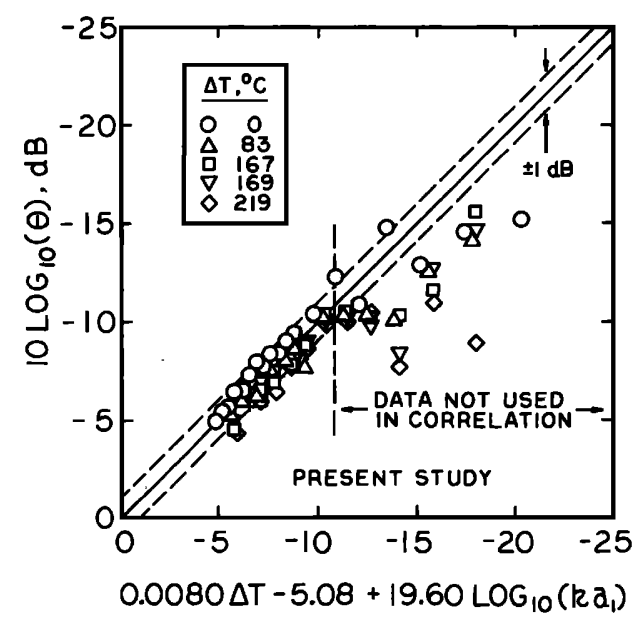

FIG. 14. Correlation of the radiation resistances from the present study using Eq. (4). the present experiment that were not used to formulate the correlation (open symbols in Fig. 8) are somewhat grouped anyway (symbols to the right of the vertical dashed line in Fig. 14). This tends to verify the hypothesis that the lowfrequency behavior of these data is not random but rather is a measure of the resistance as modified by the presence of the insulated plate $4 \frac{1}{3}$ diam from the open end of the duct.

\section{DISCUSSION}

The behavior of the ensemble of data used in this study, represented by Eq. (4), may be generalized as follows: the shape of the specific radiation resistance versus dimensionless wavenumber curve, characterized by the slope coeffcient $B$, is insensitive to the temperature difference between the gas flowing from the duct and the surrounding air, but the level of this curve, characterized by the intercept coefficient $A$, increases with this temperature difference. What is the physical interpretation of this result, and how may it be " explained?

The acoustic field is mostly oscillatory near the open end of the duct. Thus the acoustic energy associated with the nearfield is mostly that stored in the reactive component of the radiation impedance. As Cummings ${ }^{2}$ has suggested, this probably explains why the radiation reactance is not sensitive to temperature: it is mostly determined by the oscillation of a mass of fluid in the vicinity of the open end whose temperature is near that of the gas in the duct. It is in the acoustic farfield that this oscillatory motion produces waves which propagate away from the end of the duct, and thus it is there that the radiation resistance is mostly determined.

The preceding remarks suggest that the radiation resistance might be expected to be more sensitive to the surrounding air temperature than to the gas temperature at the duct exit. Indeed, Fricker and Roberts ${ }^{1}$ also presented their measured impedances as a function of a dimensionless wavenumber based on an arbitrary fixed temperature of $0^{\circ} \mathrm{C}$. They found that this frequency parameter seemed to collapse their resistance results. It is probably significant that $0{ }^{\circ} \mathrm{C}$ corresponds to a speed of sound in air that is only $4 \%$ below the value corresponding to $20^{\circ} \mathrm{C}$, which was the surrounding air temperature for their experiment. This implies, as they conclude, that presentation of resistance data as a function of a dimensionless wavenumber based on the gas temperature at the duct exit introduces an artificial temperature dependence by shifting the data along the $k a_{1}$ axis by a factor of $\sqrt{T_{a} / T_{e}}$. If, as the present correlation indicates, the shape of the curve does not change, this shift would be equivalent to an increase in the radiation resistance at a given value of the dimensionless wavenumber.

The possibility, suggested by Fricker and Roberts' results, that the increase in radiation resistance with the gas exit temperature observed in Figs. 1(a), 2(a), and 5(a) is nothing more than a consequence of the manner in which the results are presented, may be dismissed as follows. If this were the case, then the correct form of the correlation would be

$$
\log _{10}(\theta)=A_{0}+B_{0} \log _{10}\left(k_{a} a_{1}\right)
$$

where $A_{0}$ and $B_{0}$ correspond to the values of $A$ and $B$ when 
TABLE I. Values of the parameter $R$ defined by Eq. (7) for the range of conditions leading to the correlation represented by Eq. (4).

\begin{tabular}{|c|c|}
\hline $\begin{array}{l}\text { Fricker and Roberts }{ }^{1} \text { : } \\
\qquad \Delta T\end{array}$ & $\begin{array}{c}T_{a}=291 \mathrm{~K} \\
R\end{array}$ \\
\hline 282 & -0.78 \\
\hline 382 & -0.86 \\
\hline 482 & -0.93 \\
\hline 582 & -0.98 \\
\hline 682 & -1.06 \\
\hline 782 & -1.13 \\
\hline 882 & -1.19 \\
\hline 982 & -1.25 \\
\hline $\begin{array}{c}\text { Cummings } \\
\qquad T\end{array}$ & $\begin{array}{c}T_{a}=293 \mathrm{~K} \\
R\end{array}$ \\
\hline 30 & -0.58 \\
\hline 80 & -0.62 \\
\hline 130 & -0.67 \\
\hline 180 & -0.71 \\
\hline 230 & -0.75 \\
\hline 280 & -0.78 \\
\hline $\begin{array}{c}\text { Present study: } \\
\Delta T\end{array}$ & $\begin{array}{c}T_{a}=290 \mathrm{~K} \\
R\end{array}$ \\
\hline 83 & -0.62 \\
\hline 167 & -0.69 \\
\hline 169 & -0.69 \\
\hline 219 & -0.73 \\
\hline
\end{tabular}

$\Delta T=0$, and $k_{a}$ is the wavenumber based on the ambient temperature. If Eq. (4) is also expressed in terms of the frequency parameter $k_{a} a_{1}$, there results

$$
\begin{aligned}
\log _{10}(\theta)= & 0.00080 \Delta T-0.508+0.980 \log _{10}\left(T_{a} / T_{e}\right) \\
& +1.960 \log _{10}\left(k_{a} a_{1}\right) .
\end{aligned}
$$

In writing Eq. (6), use has been made of the identity

$$
k a_{1}=\sqrt{T_{a} / T_{e}} k_{a} a_{1},
$$

and the reasonable approximation $B \sim B_{0}=1.960$ has been invoked. Given that Eq. (6) represents the collective behavior of the data from the three experiments, then Eq. (5) is valid only if

$$
R \equiv \frac{0.00080 \Delta T}{0.980 \log _{10}\left(T_{a} / T_{e}\right)}=-1.0 .
$$

The parameter $R$ defined by Eq. (7) is tabulated in Table I for the range of conditions of the data used in developing the correlation represented by Eq. (4). The rather wide range of values of $R$ in this table demonstrates clearly that Eq. (5) is not generally valid, and thus that the temperature effect on the radiation resistance is not entirely due to the manner in which the results are presented. However, it is easy to see why Fricker and Roberts conclude that Eq. (5) is valid; the values of $\boldsymbol{R}$ for the range of temperatures of their study are reasonably close to -1.0 , especially in the midrange of their duct exit temperatures.

The physical explanation of the success of Eq. (4) in correlating the temperature dependent radiation resistances is elusive. It is clear, however, based on the partial success of Eq. (5), which is independent of the duct exit temperature, that the characteristic temperature which determines the radiation resistance does not vary greatly with the duct exit temperature. This is consistent with the idea that the radiation resistance is mostly determined in a region far from the duct exit for which the characteristic temperature is much more sensitive to that of the surroundings than to that of the gas flowing from the duct. In this scenario, the characteristic temperature would also be a function of frequency because the distance from the duct exit to where the resistance mechanism begins to manifest itself is measured in wavelengths. This suggests that the best correlation would be one of the form of Eq. (1) where $A$ and $B$ are constants and the wavenumber $k$ is based on a characteristic temperature which depends on the duct exit temperature, the surrounding air temperature, the frequency, and the geometry. To date we have not been able to find a suitable empirical expression for such a characteristic temperature. Though a correlation of this latter type would be more acceptable from a pedagogical point of view, the one represented by Eq. (4) is easier to use and thus more practical.

\section{CONCLUSIONS}

An experimentally based correlation has been developed for predicting the radiation resistance of thick-walled circular ducts exhausting hot gases into relatively cold surroundings. Wall thickness effects were evidently masked by other factors not systematically sampled in the study, and thus are not included in the correlation. However, the correlation, which is valid for moderate ambient temperatures (near "room temperature") and for duct exit temperatures up to $1000^{\circ} \mathrm{C}$, predicts the measured radiation resistance to within about $\pm 1.0 \mathrm{~dB}$ for a range of radius ratios between 0.60 and 0.93 . Thus, the inability of the correlation to predict wall thickness effects does not seriously limit its value. Finally, the correlation has been used to demonstrate that the temperature dependence of the radiation resistance is real and is not, as suggested by the results of an earlier study, ${ }^{1}$ merely a consequence of presenting the results in terms of a temperature dependent wavenumber.

\section{ACKNOWLEDGMENTS}

This work was partially supported by a contract with the Gas Turbine Division of the General Electric Company in Schenectady, New York, and by NASA Grant NAG3124 from the Lewis Research Center in Cleveland. The first author is also indebted to 1'Office National d'Etudes et de Recherches Aérospatiales in Châtillon-sous-Bagneux, France, where he spent the 1982-83 academic year, for providing the environment and other resources required to write this paper.

\footnotetext{
${ }^{1}$ N. Fricker and C. A. Roberts, "The Measurement of the Acoustic Radiation Impedance of the Open End of a Thick Walled Tube with Hot Flow," Acustica 38 (2), 124-130 (1977).

${ }^{2} A$. Cummings, "High Temperature Effects on the Radiation Impedance of an Unflanged Duct Exit," J. Sound Vib. 52(2), 299-304 (1977).

${ }^{3} Y$. Ando, "On the Sound Radiation from Semi-Infinite Circular Pipe of
} 
Certain Wall Thickness," Acustica 22, 219-225 (1969-70)

4J. R. Mahan and J. D. Jones, "Recovery of Acoustic Source Structure in a Long Turbulent Burner from Far-Fịeld Sound Spectra," AIAA-83-0763, AIAA 8th Aeroacoustics Conference, Atlanta, GA, 11-13 April 1983.

'P. M. Morse and K. U. Ingard, Theoretical Acoustics (McGraw-Hill, New York, 1962), pp. 469-471.
York, 1962), pp. 469-471.

'J. R. Mahan and J. M. Kasper, "Influence of Heat Release Distribution on the Acoustic Response of Long Burners," ASME-79-DET-31, ASME Design Engineering Technical Conference, St. Louis, MO, 10-12 September 1979. 